\title{
Associação entre insatisfação com a imagem corporal e aptidão física relacionada à saúde em adolescentes
}

\author{
Association between dissatisfaction with body image \\ and health-related physical fitness among adolescents
}

Gaia Salvador Claumann (https://orcid.org/0000-0002-3859-9106) ${ }^{1}$

Maria Fernanda Laus (https://orcid.org/0000-0002-4947-1642) ${ }^{2}$

Érico Pereira Gomes Felden (https://orcid.org/0000-0002-6924-122X) ${ }^{1}$

Diego Augusto Santos Silva (https://orcid.org/0000-0002-0489-7906) ${ }^{3}$

Andreia Pelegrini (https://orcid.org/0000-0001-8862-9636) ${ }^{1}$
${ }^{1}$ Programa de PósGraduação em Ciências do Movimento Humano, Centro de Ciências da Saúde e do Esporte, Universidade do Estado de Santa Catarina. R. Pascoal Simone 358, Coqueiros. 88080-350 Florianópolis SC Brasil. gaiasclaumann@ hotmail.com

${ }^{2}$ Laboratório de Nutrição e Comportamento,

Departamento de Psicologia, Faculdade de Filosofia

Ciências e Letras de Ribeirão Preto, Universidade de São Paulo. Ribeirão Preto SP Brasil.

${ }^{3}$ Programa de Pós-

Graduação em Educação

Física, Universidade

Federal de Santa Catarina.

Florianópolis SC Brasil.

\begin{abstract}
The scope of this study was to estimate the prevalence of dissatisfaction with body image and verify the association between dissatisfaction with thinness or with overweight and health-related physical fitness components among adolescents. Participants included 1058 adolescents (570 girls and 488 boys), with mean age of $16.3( \pm 1.0)$ years. The investigated variables were dissatisfaction with body image (Figure Rating Scale), and health-related physical fitness: body fat (skin folds), muscle strength (handgrip strength) and cardiorespiratory fitness (mCAFT test). The association of body image and the health-related physical fitness variables was tested by Multinomial Regression analysis. The prevalence of body dissatisfaction was $75.2 \%$ (girls $=79.5 \%$; boys $=$ 70.3\%). Girls and boys with high body fat were less likely to be dissatisfied with thinness and more likely to be dissatisfied with overweight. Those who needed to improve muscle strength and cardiorespiratory fitness were more likely to be dissatisfied with thinness and with overweight, respectively. In conclusion, physical fitness was associated with body image, with the adolescents with less healthy conditions of the components being more dissatisfied.
\end{abstract}

Key words Body image, Physical fitness, Adolescent health
Resumo O objetivo deste estudo foi estimar a prevalência de insatisfação com a imagem corporal e verificar a associação entre a insatisfação pela magreza e pelo excesso de peso e componentes da aptidão física relacionada à saúde em adolescentes. Participaram 1.058 adolescentes (570 moças e 488 rapazes), com média de idade de $16,3( \pm 1,0)$ anos. As variáveis investigadas foram a insatisfação com a imagem corporal (escala de silhuetas), e as relativas à aptidão fisica relacionada à saúde: adiposidade corporal (dobras cutâneas), força muscular (preensão manual) e aptidão cardiorrespiratória (teste mCAFT). A associação entre a imagem corporal e as variáveis de aptidão física foi testada por meio da Regressão Multinomial. A prevalência de insatisfação corporal foi de $75,2 \%($ moças $=79,5 \%$; rapazes $=70,3 \%)$. Moças e rapazes com adiposidade corporal elevada tiveram menor chance de insatisfação pela magreza e maior chance de insatisfação pelo excesso de peso. Os que necessitavam melhorar a força muscular e a aptidão cardiorrespiratória tiveram maior chance de insatisfação pela magreza e pelo excesso de peso, respectivamente. Conclui-se que a aptidão física relacionada à saúde esteve associada à imagem corporal, sendo que os adolescentes com niveis menos saudáveis dos diferentes componentes foram os mais insatisfeitos.

Palavras-chave Imagem corporal, Aptidão física, Saúde do adolescente 


\section{Introdução}

Estudos têm verificado que os diferentes componentes da aptidão física relacionada à saúde (como adiposidade corporal, força muscular e, principalmente, aptidão cardiorrespiratória) apresentam relação com o autoconceito ${ }^{1-4}$, a autoestima global e física ${ }^{5}$ e a percepção de aceitação social e da aparência física ${ }^{6} \mathrm{em}$ crianças e adolescentes. As evidências de tais investigações consistentemente apontam que indivíduos que possuem melhores níveis de aptidão física são mais positivos a respeito dos aspectos citados, sendo a relação contrária também verdadeira.

Esses aspectos (como autoconceito e autoestima), por sua vez, estão intimamente ligados à imagem corporal ${ }^{7-10}$. Dessa forma, seria adequado supor que os componentes da aptidão física relacionada à saúde também apresentam relação com a imagem corporal. Entretanto, os resultados dos poucos estudos identificados na literatura, que investigaram o relacionamento da aptidão cardiorrespiratória e força muscular com a imagem corporal, são divergentes ${ }^{11-17}$. Hausenblas et al. ${ }^{12}$ verificaram que menores níveis de aptidão cardiorrespiratória estiveram associados à insatisfação com a imagem corporal, mas essa relação perdeu significância ao controlar os efeitos do status do peso na insatisfação. De modo semelhante, os resultados encontrados por Olvera et al. ${ }^{15}$, relativos à menor satisfação, deixaram de ser significativos quando foram incluídos no modelo, junto à aptidão cardiorrespiratória, o status do peso e o percentual de gordura. Por sua vez, em estudo longitudinal realizado com crianças, menores níveis de aptidão cardiorrespiratória foram preditores de insatisfação corporal, mesmo após ajustado pelo percentual de gordura corporal ${ }^{14}$. Ainda, no estudo de Greenleaf et al. ${ }^{11}$, após controle pelo nível econômico, moças e rapazes com níveis de aptidão cardiorrespiratória adequados à saúde apresentaram maior satisfação corporal. As adolescentes avaliadas por Mitchell et al. ${ }^{13}$ que apresentaram maior aptidão cardiorrespiratória também se mostraram mais satisfeitas com seus corpos.

Dentre os estudos levantados, diferente dos demais, o de Riiser et al. ${ }^{16}$ não encontrou nenhuma relação entre a aptidão cardiorrespiratória e a imagem corporal de adolescentes com sobrepeso e obesidade, em nenhum nível. Por último, a pesquisa de Schubert et al. ${ }^{17}$ foi a única encontrada conduzida em amostra de adolescentes brasileiros e considerou além da aptidão cardiorrespiratória a força/resistência abdominal. Em ambos os componentes da aptidão física o não atendimento aos critérios de saúde esteve associado à insatisfação corporal. Porém, após ajuste pelo status do peso, flexibilidade e força de membros superiores, a associação com a força/resistência abdominal perdeu significância, mantendo-se apenas para a aptidão cardiorrespiratória.

No entanto, quando considerada a quantidade de gordura corporal, dentre os estudos que se dedicaram a investigar sua relação com a imagem corporal, parece existir consenso de que há maior probabilidade de indivíduos com elevada adiposidade corporal serem insatisfeitos com seu corpo e aparência ${ }^{14,15,18-21}$.

Diante do exposto, percebe-se a necessidade de maior compreensão e aprofundamento sobre como a aptidão física se relaciona com a imagem corporal em adolescentes, tendo em vista que são escassas as informações disponíveis sobre essa população. Tal conhecimento é de extrema importância, pois os componentes da aptidão física relacionada à saúde são fatores modificáveis, que podem ser melhorados com o objetivo de que sejam melhoradas também as percepções e os sentimentos das pessoas sobre seus corpos, evitando possíveis prejuízos à sua saúde física e mental decorrentes da insatisfação corporal. Dessa forma, o presente estudo teve como objetivo estimar a prevalência de insatisfação com a imagem corporal e verificar a associação entre a insatisfação pela magreza e pelo excesso de peso e componentes da aptidão física relacionada à saúde em adolescentes. É esperado que a maioria dos adolescentes estejam insatisfeitos com sua imagem corporal e que aqueles com níveis elevados de adiposidade corporal e baixos níveis de aptidão cardiorrespiratória estejam mais insatisfeitos pelo excesso de peso, enquanto aqueles com baixos níveis de força muscular estejam mais insatisfeitos pela magreza.

\section{Métodos}

O presente estudo faz parte do macroprojeto intitulado "Guia Brasileiro de Avaliação da Aptidão Física Relacionada à Saúde e Hábitos de Vida Etapa I", aprovado pelo Comitê de Ética em Pesquisa com Seres Humanos da Universidade Federal de Santa Catarina.

\section{População e amostra}

Constituíram a população deste estudo os adolescentes, de ambos os sexos, com idades de 
15 a 19 anos, matriculados no ensino médio em escolas públicas estaduais de São José, no ano de 2014. O município localiza-se no estado de Santa Catarina, na região Sul do Brasil e faz divisa territorial com Florianópolis, capital do estado, junto com a qual forma a região metropolitana mais populosa de Santa Catarina. De acordo com a Secretaria Estadual de Educação de Santa Catarina, em 2014, 5.182 estudantes estavam matriculados no ensino médio, distribuídos em 170 turmas nas 11 escolas elegíveis. Desse quantitativo de alunos, $74,8 \%$ eram do turno diurno (manhã, tarde ou integral).

Para determinação do tamanho da amostra, considerando-se a população finita de 5.182 estudantes, foram utilizados os procedimentos sugeridos por Luiz e Magnanini22 ${ }^{22}$ adotando-se nível de confiança de 5\%, erro tolerável de cinco pontos percentuais, prevalência de 50\% (desfecho não conhecido), e efeito de delineamento de 1,5 . Por se tratar de um estudo de associação, acrescentou-se $20 \%$ para minimizar eventuais perdas e recusas ao estudo e mais $20 \%$ para o controle de possíveis variáveis de confusão. A partir disso, o tamanho amostral necessário seria de 751 estudantes.

O processo de seleção amostral foi determinado em dois estágios: estratificado por escolas públicas estaduais de ensino médio e por conglomerado de turmas, considerando o turno de estudo e o ano escolar. Com exceção das escolas estaduais com Educação de Jovens e Adultos (EJA) e que atendiam alunos com algum tipo de deficiência intelectual, as demais foram consideradas elegíveis para a participação no estudo. No primeiro estágio a densidade da escola (tamanho: pequenas, com menos de 200 alunos; médias, com 200 a 499 alunos; e grande, com 500 estudantes ou mais) foi adotada como critério de estratificação. No segundo estágio foram considerados o turno de estudo (manhã, tarde, noite e integral) e o ano escolar (primeiro, segundo e terceiro ano).

\section{Procedimentos para coleta de dados}

A coleta de dados ocorreu no segundo semestre de 2014 (agosto à novembro), nas instalações das próprias escolas sorteadas e que aceitaram participar da pesquisa, em dias e horários agendados com os responsáveis. Inicialmente, os pesquisadores fizeram contato com os estudantes das turmas sorteadas para explicar os objetivos e a importância do estudo e convidá-los a participar do mesmo. Nesse momento, os estudantes re- ceberam o Termo de Consentimento Livre e Esclarecido (TCLE), o qual deveria ser levado para casa, para ser apresentado aos seus responsáveis e assinado caso o estudante aceitasse participar voluntariamente e seus responsáveis o autorizassem (para estudantes com idade inferior a 18 anos). Aqueles que possuíam 18 anos de idade ou mais poderiam assinar seu próprio TCLE. Além disso, todos os estudantes (independentemente da idade), receberam o Termo de Assentimento para ser assinado por eles próprios.

Em um segundo momento, os pesquisadores retornaram às turmas para recolhimento do TCLE e Termo de Assentimento e para dar início aos procedimentos de coleta de dados com os estudantes que aceitaram e receberam, quando necessário, autorização para participar do estudo. Foram realizadas três etapas, a primeira envolveu a aplicação, em sala de aula, do questionário composto por seções demográficas (sexo e idade) e insatisfação com a imagem corporal. Em seguida, em ambiente reservado, disponibilizado pela escola, foram obtidas informações sobre a maturação sexual e aferidas as medidas de dobras cutâneas, individualmente. Os testes de aptidão física relacionada à saúde (força muscular e aptidão cardiorrespiratória) foram realizados nos ginásios das escolas (quando disponíveis), ou na própria sala de aula, a qual foi organizada de maneira apropriada para a condução dos mesmos.

\section{Critérios de inclusão e exclusão}

Foram incluídos aqueles que aceitaram realizar os procedimentos voluntariamente, que assinaram o Termo de Assentimento e apresentaram o TCLE assinado por seus responsáveis (idade < 18 anos) ou por eles mesmos (idade $\geq 18$ anos). Gestantes e adolescentes que possuíam alguma deficiência física que os impedisse de realizar os testes de aptidão física não foram incluídos e receberam esclarecimentos sobre o porquê de tal decisão. Foram excluídos das análises de dados os indivíduos que possuíam idade inferior a 15 anos ou superior a 19 anos, além daqueles que não responderam às questões da variável dependente.

\section{Variáveis do estudo}

\section{Variável dependente}

A insatisfação com a imagem corporal foi avaliada por meio da escala de silhuetas de Stunkard et al. ${ }^{23}$. Os adolescentes indicaram, dentre as nove figuras possíveis para cada sexo, a silhueta que consideraram representar sua for- 
ma física atual (Silhueta Real - SR) e a silhueta que representava a forma como gostariam de se parecer, aproximadamente (Silhueta Ideal - SI). A partir disso foram classificados em satisfeitos $(\mathrm{SR}=\mathrm{SI})$, insatisfeitos pela magreza $(\mathrm{SR}<\mathrm{SI})$ e insatisfeitos pelo excesso de peso (SR $>$ SI).

\section{Variáveis independentes}

Para a avaliação da adiposidade corporal foram aferidas as medidas de dobras cutâneas das regiões do tríceps e subescapular, conforme padronização da International Society for the Advancement of Kinanthropometry ${ }^{24}$, utilizando-se adipômetro científico da marca Cescorf ${ }^{\circledR}$ com resolução de $0,1 \mathrm{~mm}$. As dobras cutâneas foram mensuradas em duplicata e no caso de diferença entre a primeira e a segunda medida em mais de $5 \%$, foi realizada uma terceira medida. As medidas foram realizadas por um único avaliador, sendo calculado o erro técnico das mesmas. Para obter um único valor de cada dobra, nos casos em que apenas duas medidas foram mensuradas, calculou-se a média entre as mesmas, já entre os casos em que foram mensuradas três medidas, utilizou-se a mediana. Realizou-se o somatório das duas dobras cutâneas ( $\Sigma 2 \mathrm{DC})$, a partir do qual os adolescentes foram classificados em baixa, normal e elevada adiposidade corporal, conforme os pontos de corte estabelecidos para cada sexo pela American Alliance for Health, Physical Education, Recreation and Dance ${ }^{25}$. Os adolescentes com adiposidade corporal elevada formaram a categoria "adiposidade corporal elevada" e, em virtude da reduzida prevalência de indivíduos com baixa adiposidade (17 rapazes), esses foram agrupados aos que apresentaram adiposidade normal, constituindo a categoria "adiposidade corporal adequada".

A força de preensão manual, mensurada por dinamômetro da marca Saehan ${ }^{\circledR}$, o qual é válido, confiável e comparável ao dinamômetro Jamar ${ }^{\circledR}$ (considerado "padrão ouro") ${ }^{26}$, foi utilizada para avaliação da força muscular. Os adolescentes realizaram a medida duas vezes em cada uma das mãos, alternadamente. Realizou-se somatório do maior valor encontrado $(\mathrm{em} \mathrm{kg})$ para a mão esquerda e direita, a partir do qual os adolescentes foram classificados, considerando-se as zonas de benefícios à saúde, em "excelente", "muito bom", "bom", "regular" e "necessita melhorar"24. As categorias excelente, muito bom e bom foram agrupadas e consideradas como "boa força muscular".

A aptidão cardiorrespiratória foi avaliada por meio do Modified Canadian Aerobic Fitness Test (mCAFT), validado a partir da calorimetria indi- reta para adolescentes, adultos e idosos ${ }^{27,28}$. Após a realização de todos os procedimentos recomendados pela Canadian Society for Exercise Physiology os adolescentes foram classificados nas seguintes categorias relacionadas aos benefícios à saúde: "excelente", "muito boa", "boa", "regular" e "necessita melhorar" ${ }^{24}$. Nenhum adolescente apresentou aptidão cardiorrespiratória excelente. As categorias muito boa e boa foram agrupadas e consideradas como "boa aptidão cardiorrespiratória”.

\section{Variáveis de controle}

A idade em anos completos (15, 16, 17, 18 e 19 anos) e a maturação sexual foram utilizadas como variáveis de controle nas análises do presente estudo, devido à forte relação das mesmas tanto com a imagem corporal quanto com a aptidão física ${ }^{29,30}$. A maturação sexual foi avaliada por meio do instrumento elaborado pelo Departamento de Nutrição da Universidade Federal de Santa Catarina ${ }^{31}$, que consiste em figuras adaptadas a partir das fotografias de Tanner (1962), correspondentes à cinco estágios de desenvolvimento para cada característica sexual secundária (mamas e pelos pubianos para o sexo feminino, órgãos genitais e pelos pubianos para o sexo masculino). No presente estudo foram considerados para classificação do estágio de maturação apenas os pelos pubianos, para ambos os sexos. Após explicação do pesquisador sobre o instrumento, cada adolescente, por meio de autoavaliação, indicou o número do estágio que mais se aproximava do desenvolvimento do seu corpo naquele momento. Os adolescentes foram classificados em pré-púberes (estágio 1), púberes (estágios 2, 3 e 4) e pós-púberes (estágio 5 ).

\section{Análise estatística}

Foram realizadas análises descritivas (média, desvio padrão, distribuição de frequências) e inferenciais. O qui-quadrado foi utilizado para identificar possíveis associações entre o sexo e as variáveis idade, maturação sexual, adiposidade corporal, força muscular, aptidão cardiorrespiratória e imagem corporal. A regressão logística multinomial foi utilizada para testar as associações entre a insatisfação com a imagem corporal e os componentes da aptidão física relacionada à saúde, com estimativa de odds ratio e os respectivos intervalos de confiança. A categoria de referência adotada para a variável dependente foi "satisfeitos com a imagem corporal". Foram conduzidas análises brutas e ajustadas. Nas análises 
bivariadas, cada componente da aptidão física relacionada à saúde foi inserido isoladamente. Nas análises multivariadas, os componentes da aptidão física relacionada à saúde foram ajustados por idade e maturação sexual, variáveis selecionadas de acordo com a literatura ${ }^{29,30}$. A qualidade do ajuste dos modelos foi analisada pelo quiquadrado de Pearson. Todas as análises estatísticas foram realizadas no software The Statistical Package for the Social Sciences (SPSS), versão 20.0. O nível de significância estabelecido em todas as análises foi de $5 \%$.

\section{Resultados}

A amostra final do presente estudo foi constituída por 1.058 adolescentes (570 moças e 488 rapazes), com média de idade de 16,3 $( \pm 1,0)$ anos. As demais características dos participantes, de acordo com as variáveis investigadas, são apresentadas na Tabela 1 .

A prevalência de insatisfação com a imagem corporal na amostra foi de 75,2\% (79,5\% das moças e $70,3 \%$ dos rapazes, $\mathrm{p}=0,001$ ), sendo que $39,5 \%$ dos adolescentes estavam insatisfeitos pelo excesso de peso e 35,7\% pela magreza. Na Figura 1 são apresentadas as proporções, estratificadas por sexo, de adolescentes satisfeitos, insatisfeitos pelo excesso de peso e pela magreza. Observou-se maior proporção de satisfeitos e insatisfeitos pela magreza no sexo masculino, e maior proporção de insatisfeitos pelo excesso de peso no sexo feminino $(\mathrm{p}<0,001)$.

Moças com adiposidade corporal elevada apresentaram menor chance de insatisfação pela magreza $(\mathrm{OR}=0,36$; IC95\% $=0,16-0,86)$ e, por outro lado, maior chance de insatisfação pelo excesso (OR $=6,54 ; \mathrm{IC} \%=3,58-11,96)$. Aquelas que necessitavam melhorar a força muscular tiveram maior chance de estarem insatisfeitas pela magreza $(\mathrm{OR}=2,19$; IC95\% = 1,05-4,55), enquanto as que necessitavam melhorar a aptidão cardiorrespiratória apresentaram maior chance de insatisfação pelo excesso de peso $(\mathrm{OR}=2,54$; IC95\% = 1,08-5,99) (Tabela 2).

Assim como observado no sexo feminino, os rapazes com adiposidade corporal elevada apresentaram menor chance de insatisfação pela magreza $(\mathrm{OR}=0,30 ; \mathrm{IC} 95 \%=0,12-0,77)$ e maior chance de insatisfação pelo excesso de peso (OR $=15,30$; IC95\% = 7,66-30,57). Os que necessi-

Tabela 1. Características dos adolescentes do ensino público estadual do município de São José-SC. 2014.

\begin{tabular}{lrrrr}
\hline \multicolumn{1}{c}{ Variáveis } & $\begin{array}{c}\text { Amostra total } \\
\mathbf{n}(\%)\end{array}$ & $\begin{array}{c}\text { Sexo feminino } \\
\mathbf{n}(\%)\end{array}$ & $\begin{array}{c}\text { Sexo masculino } \\
\mathbf{n}(\%)\end{array}$ & p-valor $^{*}$ \\
\hline Idade & & & & 0,008 \\
15 anos & $293(27,7)$ & $170(29,8)$ & $123(25,2)$ & \\
16 anos & $313(29,6)$ & $171(30,0)$ & $142(29,1)$ & \\
17 anos & $330(31,2)$ & $182(31,9)$ & $148(30,3)$ & \\
18 anos & $95(9,0)$ & $37(6,5)$ & $58(11,9)$ & \\
19 anos & $27(2,6)$ & $10(1,8)$ & $17(3,5)$ & \\
Maturação sexual & & & & \\
$\quad$ Pré-púbere & $71(6,7)$ & $35(6,2)$ & $36(7,4)$ & \\
Púbere & $842(80,0)$ & $484(85,5)$ & $358(73,7)$ & \\
$\quad$ Pós-púbere & $139(13,2)$ & $47(8,3)$ & $92(18,9)$ & \\
Adiposidade corporal & & & & \\
Adequada & $614(70,4)$ & $296(64,6)$ & $318(76,8)$ & \\
Elevada & $258(29,6)$ & $162(35,4)$ & $96(23,2)$ & \\
Força muscular & & & & \\
$\quad$ Necessita melhorar & $539(62,0)$ & $270(59,0)$ & $269(65,3)$ & \\
Regular & $165(19,0)$ & $85(18,6)$ & $80(19,4)$ & \\
Boa & $166(19,1)$ & $103(22,5)$ & $63(15,3)$ & \\
Aptidão cardiorrespiratória & & & & \\
Necessita melhorar & $499(60,5)$ & $253(58,2)$ & $246(63,1)$ & \\
Regular & $222(26,9)$ & $136(31,3)$ & $86(22,1)$ & \\
Boa & $104(12,6)$ & $46(10,6)$ & $58(14,9)$ &
\end{tabular}

n: frequência absoluta; \%: frequência relativa. ${ }^{\star}$ Qui-quadrado. 


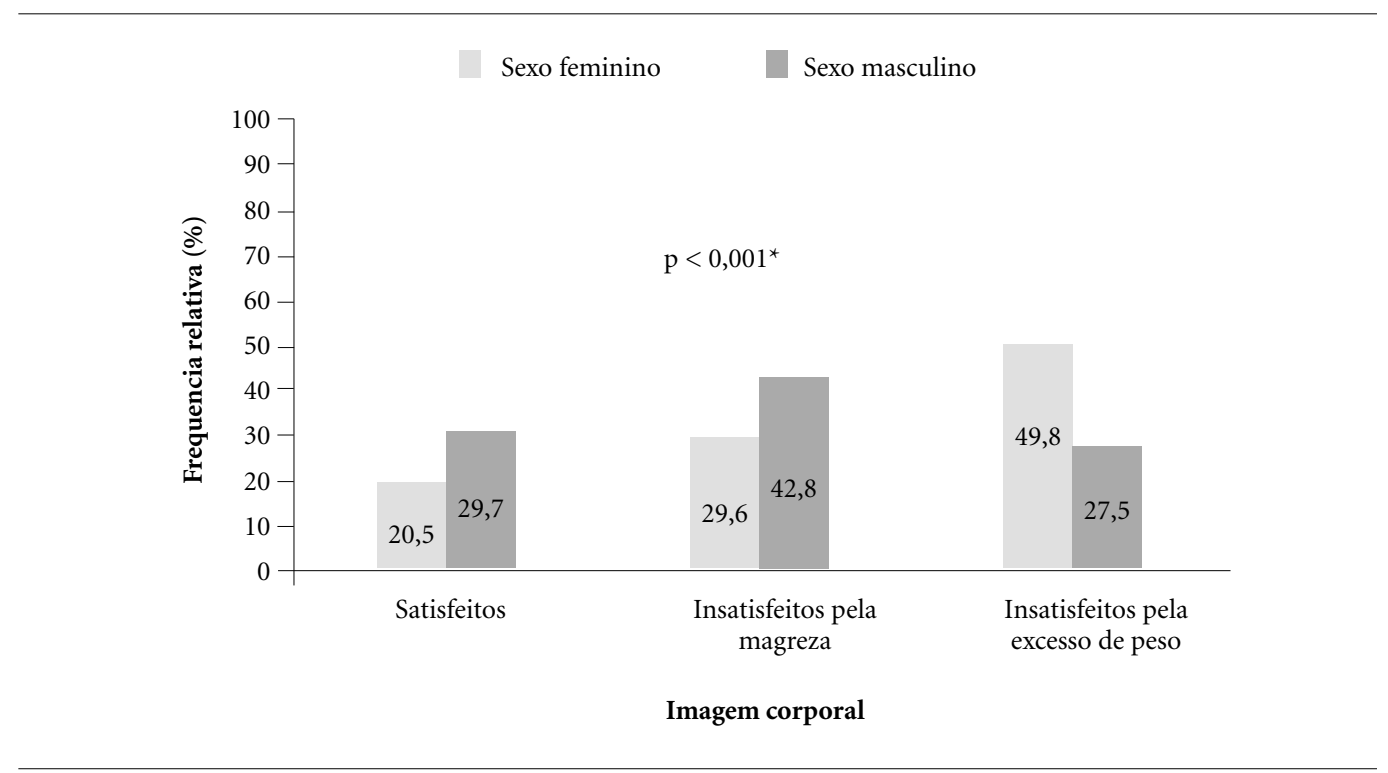

Figura 1. Insatisfação com a imagem corporal dos adolescentes do ensino público estadual do município de São José-SC, estratificado por sexo. 2014.

${ }^{\star}$ Qui-quadrado.

Tabela 2. Associação entre insatisfação com a imagem corporal e diferentes componentes da aptidão física relacionada à saúde em adolescentes do sexo feminino. São José-SC. 2014.

\begin{tabular}{|c|c|c|c|c|c|c|}
\hline \multirow{3}{*}{ Variável } & \multirow{3}{*}{$\%$} & \multirow{2}{*}{\multicolumn{2}{|c|}{$\begin{array}{c}\text { Insatisfação pela magreza } \\
\text { OR }(\text { IC95\%)* }\end{array}$}} & \multirow{3}{*}{$\%$} & \multirow{2}{*}{\multicolumn{2}{|c|}{$\begin{array}{c}\text { Insatisfação pelo excesso de peso } \\
\text { OR }(\mathrm{IC} 95 \%)^{\star} \\
\end{array}$}} \\
\hline & & & & & & \\
\hline & & Bruta & Ajustada & & Bruta & Ajustada \\
\hline \multicolumn{7}{|l|}{ Adiposidade corporal } \\
\hline Elevada & 7,4 & $0,37(0,16-0,86)$ & $0,37(0,16-0,86)$ & 58,9 & $6,71(3,68-12,23)$ & $6,54(3,58-11,96)$ \\
\hline Adequada & 92,6 & 1 & 1 & 41,1 & 1 & 1 \\
\hline \multicolumn{7}{|l|}{ Força muscular } \\
\hline Necessita melhorar & 69,1 & $2,00(0,98-4,13)$ & $2,19(1,05-4,55)$ & 53,4 & $1,02(0,55-1,88)$ & $1,04(0,56-1,94)$ \\
\hline Boa & 17,6 & $1,48(0,61-3,58)$ & $1,50(0,61-3,67)$ & 26,3 & $1,44(0,69-3,03)$ & $1,46(0,69-3,09)$ \\
\hline Regular & 13,2 & 1 & 1 & 20,3 & 1 & 1 \\
\hline \multicolumn{7}{|l|}{$\begin{array}{l}\text { Aptidão } \\
\text { cardiorrespiratória }\end{array}$} \\
\hline Necessita melhorar & 44,2 & $0,79(0,34-1,83)$ & $0,76(0,33-1,79)$ & 69,7 & $2,52(1,09-5,84)$ & $2,54(1,08-5,99)$ \\
\hline Regular & 41,1 & $0,96(0,41-2,27)$ & $0,91(0,38-2,19)$ & 23,1 & $1,10(0,45-2,66)$ & $1,06(0,43-2,62)$ \\
\hline Boa & 14,7 & 1 & 1 & 7,2 & 1 & 1 \\
\hline
\end{tabular}

\%: frequência relativa; OR: odds ratio; IC95\%: intervalo de confiança de 95\%. ${ }^{\star}$ Regressão logística multinomial. Análises ajustadas por idade e maturação sexual.

tavam melhorar a força muscular tiveram maior chance de insatisfação pela magreza $(\mathrm{OR}=2,52$; IC95\% $=1,30-4,86)$ e aqueles que necessitavam melhorar a aptidão cardiorrespiratória apresentaram maior chance de insatisfação pelo excesso de peso $(\mathrm{OR}=3,30$; IC95\% = 1,29-8,40) (Tabela 3).

\section{Discussão}

De acordo com o que era esperado, os resultados do presente estudo demonstraram que a maioria dos adolescentes estava insatisfeita com a imagem corporal. A prevalência de insatisfação encontrada $(75,2 \%)$ foi superior às prevalências 
Tabela 3. Associação entre insatisfação com a imagem corporal e diferentes componentes da aptidão física relacionada à saúde em adolescentes do sexo masculino. São José-SC. 2014.

\begin{tabular}{|c|c|c|c|c|c|c|}
\hline \multirow{3}{*}{ Variável } & \multirow{3}{*}{$\%$} & \multirow{2}{*}{\multicolumn{2}{|c|}{$\begin{array}{c}\text { Insatisfação pela magreza } \\
\text { OR }(\text { IC95\%)* }\end{array}$}} & \multirow{3}{*}{$\%$} & \multirow{2}{*}{\multicolumn{2}{|c|}{$\begin{array}{c}\text { Insatisfação pelo excesso de peso } \\
\text { OR }(\text { IC95\%)* }\end{array}$}} \\
\hline & & & & & & \\
\hline & & Bruta & Ajustada & & Bruta & Ajustada \\
\hline \multicolumn{7}{|l|}{ Adiposidade corporal } \\
\hline Elevada & 4,4 & $0,34(0,14-0,85)$ & $0,30(0,12-0,77)$ & 66,1 & $14,61(7,39-28,86)$ & $15,30(7,66-30,57)$ \\
\hline Adequada & 95,6 & 1 & 1 & 33,9 & 1 & 1 \\
\hline \multicolumn{7}{|l|}{ Força muscular } \\
\hline Necessita melhorar & 73,6 & $2,49(1,30-4,79)$ & $2,52(1,30-4,86)$ & 64,5 & $1,63(0,81-3,29)$ & $1,58(0,78-3,21)$ \\
\hline Regular & 14,8 & $1,04(0,48-2,25)$ & $1,08(0,49-2,37)$ & 20,0 & $1,04(0,46-2,38)$ & $1,04(0,45-2,37)$ \\
\hline Boa & 11,5 & 1 & 1 & 15,5 & 1 & 1 \\
\hline \multicolumn{7}{|l|}{$\begin{array}{l}\text { Aptidão } \\
\text { cardiorrespiratória }\end{array}$} \\
\hline Necessita melhorar & 59,1 & $0,87(0,45-1,68)$ & $0,86(0,45-1,67)$ & 78,1 & $3,35(1,32-8,51)$ & $3,30(1,29-8,40)$ \\
\hline Regular & 21,6 & $0,61(0,29-1,28)$ & $0,60(0,28-1,26)$ & 15,2 & $1,25(0,43-3,60)$ & $1,25(0,43-3,60)$ \\
\hline Boa & 19,3 & 1 & 1 & 6,7 & 1 & 1 \\
\hline
\end{tabular}

verificadas em pesquisas que também utilizaram escalas de silhuetas como instrumento de avaliação (embora as escalas não tenham sido as mesmas), conduzidas em adolescentes de diferentes cidades e estados da região Sul do Brasil ${ }^{32-37}$, bem como de outras regiões do país ${ }^{38}$, podendo ser considerada elevada. Entretanto, o percentual de adolescentes que desejam ter um corpo diferente do atual é bastante semelhante ao verificado em adolescentes de Hong Kong ${ }^{39}$ e dos Emirados Árabes ${ }^{40}$, e até mesmo inferior ao de adolescentes taiwaneses ${ }^{41}$. Todos esses, por sua vez, estão inseridos em culturas orientais, o que é surpreendente pois a literatura aponta que as preocupações com o corpo são uma realidade mais comum em países de cultura ocidental ${ }^{42}$.

As análises de associação indicaram que todos os componentes da aptidão física relacionada à saúde investigados neste estudo se associaram à insatisfação com a imagem corporal. Embora tenham sido conduzidas análises separadas para moças e rapazes, em virtude de estudos prévios demonstrarem consistentemente que a imagem corporal difere entre os $\operatorname{sexos}^{32,35,36,39-41}$, os resultados encontrados foram semelhantes no sexo feminino e masculino.

Indo ao encontro da hipótese levantada, os adolescentes (de ambos os sexos) com adiposidade corporal elevada apresentaram menor chance de insatisfação pela magreza e maior chance de insatisfação pelo excesso de peso. Esses resultados corroboram os achados de Ferrari et al. ${ }^{18}$, em es- tudo conduzido com rapazes de sete a 17 anos de idade de um município da região Sul do Brasil, e podem indicar que estes indivíduos possuem percepção adequada da sua forma física, apesar de não estarem satisfeitos com isso e, portanto, devem ser orientados sobre comportamentos saudáveis para modificá-la, caso desejem.

A força muscular se associou à insatisfação pela magreza. Confirmando o que era esperado, os adolescentes que necessitavam melhorar este componente da aptidão física apresentaram maior chance de desejarem um corpo com proporções maiores do que o seu atual. Schubert et al. ${ }^{17}$ também verificaram associação entre menores níveis de força muscular e insatisfação com a imagem corporal em crianças e adolescentes, destacando-se, porém, que a forma de avaliação da força não foi a mesma da presente investigação. Para além disso, não foram encontrados estudos que investigassem associações com a insatisfação pela magreza e pelo excesso de peso, especificamente. Dessa forma, é plausível supor que o fato daqueles que possuem menor força desejarem silhuetas maiores do que as suas atuais pode estar relacionado à crença popular de que um corpo magro também é um corpo fraco e frágil, e que um maior desenvolvimento muscular necessariamente implica no aumento da força como qualidade física ${ }^{43}$.

Em relação à aptidão cardiorrespiratória, os adolescentes com níveis mais baixos desse componente apresentaram maior chance de insatisfa- 
ção pelo excesso de peso, o que também confirma a hipótese levantada. Acredita-se que a relação entre aptidão cardiorrespiratória e imagem corporal seja mediada pela quantidade de gordura corporal, pois tem sido demonstrado que maiores níveis de aptidão cardiorrespiratória se associam à baixa adiposidade corporal total e central, sendo a relação contrária também verdadeira ${ }^{44}$. Dessa forma, adolescentes mais aptos são mais prováveis de possuírem um corpo conforme os padrões sociais e, assim, demonstrarem satisfação, conforme sugerem Mitchell et al. ${ }^{13}$.

No entanto, estudos que investigaram possíveis associações entre aptidão cardiorrespiratória e imagem corporal, controlando pelo percentual de gordura, apresentaram resultados divergentes. Enquanto no estudo de Olive et al. ${ }^{14}$ crianças com menores níveis de aptidão cardiorrespiratória apresentaram maior probabilidade de insatisfação corporal, mesmo após o controle pela adiposidade corporal, as associações entre essas variáveis deixaram de ser significativas quando foram incluídos no modelo, junto à aptidão cardiorrespiratória, o status do peso e o percentual de gordura. Diante disso, parece que tanto baixos níveis de aptidão cardiorrespiratória quanto a elevada adiposidade corporal contribuem para a insatisfação corporal, mas que as relações entre essas variáveis podem ser independentes, havendo, possivelmente, mecanismos distintos que as expliquem e que ainda necessitam de investigação.

Nesse sentido, uma explicação alternativa para nosso resultado é de que indivíduos mais aptos, percebendo-se fisicamente mais capazes, podem ter sentimentos mais positivos em relação ao seu corpo e, assim, se preocuparem menos com a aparência do corpo e mais com o que ele é capaz de fazer, e vice-versa. O fato de acreditarem em sua competência física pode substituir as comparações de quão próximos estão de ter a aparência internalizada como socialmente ideal, por como seu corpo consegue realizar atividades da vida diária ou atividades físicas e esportivas, por exemplo ${ }^{13}$.

De modo geral, as evidências desta investigação reforçam os achados da literatura sobre a temática e acrescentam informações, principalmente sobre as direções das associações de diferentes componentes da aptidão física relacionada à saúde com a insatisfação com a imagem corpo- ral especificamente pela magreza ou pelo excesso de peso. A partir disso, aponta-se a importância de tais componentes da aptidão física serem considerados em programas de intervenção que tenham como objetivo a melhora da imagem corporal, além dos aspectos psicológicos que normalmente são levados em conta. Profissionais diretamente envolvidos com os adolescentes, especialmente os da saúde, devem estar atentos a tais necessidades.

Os resultados apresentados, entretanto, devem ser interpretados levando-se em consideração uma série de limitações, como por exemplo o delineamento transversal que impossibilita o estabelecimento de relação causal entre as variáveis; a amostra constituída apenas por escolares da rede pública, limitando a extrapolação dos achados aos adolescentes do ensino privado, que podem apresentar, por exemplo, características socioeconômicas e culturais diferentes que influenciam em diferentes aspectos, como em suas experiências com a imagem corporal e oportunidades de desenvolvimento da aptidão física; ainda a respeito da amostra, o estudo incluiu uma amostra superestimada em relação ao tamanho amostral necessário calculado. Isto ocorreu pois no momento da coleta de dados todos os alunos das turmas sorteadas foram convidados para participar do estudo e havia turmas com número de alunos diferente daquele fornecido pela Secretaria Estadual de Educação; outra limitação está relacionada ao uso da escala de silhuetas de Stunkard et al. ${ }^{23}$, a qual não possui validação para a população adolescente. Apesar disso, ressaltase que sua utilização com indivíduos dessa faixa etária vem sendo cada vez maior, sobretudo em estudos brasileiros ${ }^{33,36-38}$.

Em conclusão, cerca de $3 / 4$ da amostra investigada estava insatisfeita com sua imagem corporal. As associações encontradas entre imagem corporal e os componentes da aptidão física relacionada à saúde foram semelhantes entre os sexos. A adiposidade corporal elevada esteve associada à menor chance de insatisfação pela magreza e maior de insatisfação pelo excesso de peso. Menores níveis de força muscular estiveram associados à maior chance de insatisfação pela magreza, enquanto níveis mais baixos de aptidão cardiorrespiratória se associaram à maior chance de insatisfação pelo excesso de peso. 


\section{Colaboradores}

GS Claumann participou da concepção e delineamento do estudo, coleta de dados, análise e interpretação dos mesmos, redação do artigo e aprovou a versão final a ser publicada. MF Laus auxiliou na interpretação dos dados, redação do artigo, revisão crítica e aprovação da versão final a ser publicada. EPG Felden participou da análise e interpretação dos dados, revisão crítica e aprovação da versão final a ser publicada. DAS Silva participou da concepção e delineamento do estudo, análise dos dados, revisão crítica e aprovação da versão final a ser publicada. A Pelegrini participou da concepção e delineamento do estudo, análise e interpretação dos dados, revisão crítica e aprovação da versão final a ser publicada.

\section{Referências}

1. Carraro A, Scarpa S, Ventura L. Relationships between physical self-concept and physical fitness in Italian adolescents. Percept Mot Skills 2010; 110(2):522-530.

2. Casas AG, García PLR, Guillamón AR, García-Cantó E, Soto JJP, López PT. Capacidad aeróbica, estado de peso y autoconcepto en escolares de primaria. Clin Investig Arterioscler 2016; 28(1):1-8.

3. Garcia PLR, Galvez A, Canto EG, Soto JJP, Guillamon AR, Marcos LT, Lopez PJT. Relationship between the Self-Concept and Muscular Strength in Southern Spanish Children. J Psychol Psychother 2015; 5(6):1.

4. Reigal-Garrido RE, Becerra-Fernandez CC, Hernandez-Mendo A, Martin-Tamayo I. Relación del autoconcepto con la condición física y la composición corporal en una muestra de adolescentes. Anales de Psicología 2014; 30(3):1079-1085.

5. Lubans DR, Cliff DP. Muscular fitness, body composition and physical self-perception in adolescents. $J$ Sci Med Sport 2011; 14(3):216-221.

6. Vedul-Kjelsås V, Sigmundsson H, Stensdotter AK, Haga $\mathrm{M}$. The relationship between motor competence, physical fitness and self-perception in children. Child Care Health Dev 2012; 38(3):394-402.

7. Fernández-bustos J, González-Martí I, Contreras O, Cuevas R. Relación entre imagen corporal y autoconcepto físico en mujeres adolescentes. Rev Latinoam Psicol 2015; 47(1):25-33.

8. Martins DF, Nunes MFO. Noronha APP. Satisfação com a imagem corporal e autoconceito em adolescentes. Psicol Teor Prat 2008; 10(2):94-105.

9. Morin AJS, Maiano C, Marsh HW, Janosz M, Nagengast $\mathrm{B}$. The longitudinal interplay of adolescents' self-esteem and body image: A conditional autoregressive latent trajectory analysis. Multivariate Behav Res 2011; 46(2):157-201.

10. Wichstrøm L, Von Soest T. Reciprocal relations between body satisfaction and self-esteem: A large 13year prospective study of adolescents. J Adolesc 2016; 47:16-27.

11. Greenleaf CA, Petrie TA, Martin SB. Psychosocial variables associated with body composition and cardiorespiratory fitness in middle school students. Res Q Exerc Sport 2010; 81(Supl. 3):S65-S74.

12. Hausenblas HA, Symons Downs D, Fleming DS, Connaughton DP. Body image in middle school children. Eat Weight Disord ST 2002; 7(3):244-248.

13. Mitchell SH, Petrie TA, Greenleaf CA, Martin SB. Moderators of the internalization-body dissatisfaction relationship in middle school girls. Body Image 2012; 9(4):431-440.

14. Olive LS, Byrne DG, Cunningham RB, Telford RD. Effects of physical activity, fitness and fatness on children's body image: the Australian LOOK longitudinal study. Ment Health Phys Act 2012; 5(2):116-124.

15. Olvera N, McCarley K, Rodriguez AX, Noor N, Hernández-Valero MA. Body image disturbances and predictors of body dissatisfaction among hispanic and white preadolescents. J Res Adolesc 2015; 25(4):728-738.

16. Riiser K, Ommundsen Y, Smastuen MC, Londal K, Misvaer N, Helseth S. The relationship between fitness and health-related quality of life and the mediating role of self-determined motivation in overweight adolescents. Scand J Public Health 2014; 42(8):766-772. 
17. Schubert A, Januário RSB, Casonatto J, Sonoo CN. Imagem corporal, estado nutricional, força de resistência abdominal e aptidão cardiorrespiratória de crianças e adolescentes praticantes de esportes. Rev Paul Pediatr 2013; 31(1):71-76.

18. Ferrari EP, Minatto G, Berria J, Silva SF, Fidelix YL, Ribeiro RR, Santos KD, Petroski EL. Body image dissatisfaction and anthropometric indicators in male children and adolescents. Eur J Clin Nutr 2014; 69(10):1140-1144.

19. Fortes LS, Almeida SS, Ferreira MEC. Indicadores antropométricos de insatisfação corporal e de comportamentos alimentares inadequados em jovens atletas. Rev Bras Med Esporte 2013; 19(1):35-39.

20. Koyuncu M, Tok S, Canpolat AM, Catikkas F. Body image satisfaction and dissatisfaction, social physique anxiety, self-esteem, and body fat ratio in female exercisers and nonexercisers. Soc Behav Personal: Int J 2010; 38(4):561-570.

21. Martins CR, Pelegrini A, Matheus SC, Petroski EL. Insatisfação com a imagem corporal e relação com estado nutricional, adiposidade corporal e sintomas de anorexia e bulimia em adolescentes. Rev Psiquiatr Rio Gd. Sul 2010; 32(1):19-23.

22. Luiz RR, Magnanini MMF. A lógica da determinação do tamanho da amostra em investigações epidemiológicas. Cad Saude Colet 2000; 8(2):9-28.

23. Stunkard AJ, Sorensen T, Schulsinger F. Use of the Danish Adoption Register for the study of obesity and thinness. In: Kety SS, Rowland LP, Sidman RL, Matthysse SW. editors. The Genetics of Neurological and Psychiatric Disorders. New York: Raven Press; 1983. p. 115-120.

24. Canadian Society For Exercise Physiology. The Canadian Physical Activity, Fitness and Lifestyle Approach (CPAFLA). $3^{\text {th }}$ ed. Ottawa: Canadian Society for Exercise Physiology; 2003.

25. American Alliance For Health, Physical Education, Recreation And Dance (AAHPERD). Physical Best. Reston: AAHPERD; 1988.

26. Reis MM, Arantes PMM. Medida de força de preensão manual: validade e confiabilidade do dinamômetro Saehan. Fisioter Pesqui 2011; 18(2):176-181.

27. Weller JMR, Thomas SG, Corey PN, Cox MH. Prediction of maximal oxygen uptake from a modified Canadian Aerobic Fitness Test. Can J Appl Physiol 1993; 18(2):175-188.

28. Weller JMR, Thomas SG, Gledhill N, Paterson D, Quinney A. A study to validate the modified Canadian Aerobic Fitness Test. Can J Appl Physiol 1995; 20(2):211-221.

29. Klump KL. Puberty as a critical risk period for eating disorders: a review of human and animal studies. Horm Behav 2013; 64(2):399-410.

30. Ortega FB, Ruiz JR, Castillo MJ, Moreno LA, Urzanqui A, González-Gross M, Sjöström M, Gutiérrez A; AVENA Study Group. Health-related physical fitness according to chronological and biological age in adolescents. J Sports Med Phys Fitness 2008; 48(3):371-379.

31. Adami F, Vasconcelos FAG. Obesidade e maturação sexual precoce em escolares de Florianópolis - SC. Rev Bras Epidemiol 2008; 11(4):549-560.

32. Dumith SC, Menezes AMB, Bielemann RM, Petresco S, Silva ICM, Linhares RS, Amorim TC, Duarte DV, Araújo CLP, Santos JV. Insatisfação corporal em adolescentes: um estudo de base populacional. Cien Saude Colet 2012; 17(9):2499-2505.
33. Felden EPG, Claumann GS, Sacomori C, Daronco LSE, Cardoso FL, Pelegrini A. Fatores sociodemográficos e imagem corporal em adolescentes do ensino médio. Cien Saude Colet 2015: 20(11):3329-3337.

34. Fidelix YL, Minatto G, Ribeiro RR, Santos KD, Petroski EL. Dados sociodemográficos, estado nutricional e maturação sexual de escolares do sexo masculino: exposição à insatisfação com a imagem corporal. Rev Educ Fis/UEM 2013; 24(1):83-92.

35. Finato S, Rech RR, Migon P, Gavineski IC, Toni V, Halpern R. Insatisfação com a imagem corporal em escolares do sexto ano da rede municipal de Caxias do Sul, no Rio Grande do Sul. Rev Paul Pediatr 2013; 31(1):65-70.

36. Glaner MF, Pelegrini A, Cordoba CO, Pozzobon ME. Associação entre insatisfação com a imagem corporal e indicadores antropométricos em adolescentes. Rev Bras Educ Fis Esporte 2013; 27(1):129-136.

37. Pelegrini A, Coqueiro RS, Beck CC, Ghedin KD, Lopes AS, Petroski EL. Dissatisfaction with body image among adolescent students: association with socio-demographic factors and nutritional status. Cien Saude Colet 2014; 19(4):1201-1208.

38. Fidelix YL, Silva DAS, Pelegrini A, Silva AF, Petroski EL. Insatisfação com a imagem corporal em adolescentes de uma cidade de pequeno porte: associação com sexo, idade e zona de domicílio. Rev Bras Cineantropom Desempenho Hum 2011; 13(3):202-207.

39. Lai CM, Mak KK, Pang JS, Fong SS, Ho RC, Guldan GS. The associations of sociocultural attitudes towards appearance with body dissatisfaction and eating behaviors in Hong Kong adolescents. Eat Behav 2013; 14(3):320-324.

40. Schulte SJ, Thomas J. Relationship between eating pathology, body dissatisfaction and depressive symptoms among male and female adolescents in the United Arab Emirates. Eat Behav 2013; 14(2):157-160.

41. Chen LJ, Fox KR, Haase AM, Ku PW. Correlates of body dissatisfaction among Taiwanese adolescents. Asia Pac J Clin Nutr 2010; 19(2):172-179.

42. Tiggemann M. Considerations of positive body image across various social identities and special populations. Body Image 2015; 14:168-176.

43. Tatangelo GL, Ricciardelli LA. A qualitative study of preadolescent boys' and girls' body image: gendered ideals and sociocultural influences. Body Image 2013; 10(4):591-598.

44. Moliner-Urdiales D, Ruiz JR, Vicente-Rodriguez G, Ortega FB, Rey-Lopez JP, España-Romero V, Casajús JA, Molnar D, Widhalm K, Dallongeville J, GonzálezGross M, Castillo MJ, Sjöström M, Moreno LA. Associations of muscular and cardiorespiratory fitness with total and central body fat in adolescents: the HELENA Study. Br J Sports Med 2011; 45(2):101-108.

Artigo apresentado em 11/01/2017

Aprovado em 23/07/2017

Versão final apresentada em 25/07/2017 